\title{
NONLINEAR COMPONENT OF FREE VIBRATION FREQUENCY OF A PARTIALLY TENSIONED SYSTEM WITH CONSIDERATION OF ROTATIONAL STIFFNESS OF SUPPORT
}

\author{
Sebastian Uzny, Michat Osadnik \\ Institute of Mechanics and Machine Design Fundamentals, Czestochowa University of Technology \\ Częstochowa, Poland \\ uzny@imipkm.pcz.pl,mosadnik@imipkm.pcz.pl
}

Received: 15 May 2019; Accepted: 11 August 2019

\begin{abstract}
The nonlinear vibrations of a slender system subjected to Euler's load which is partially tensioned is discussed in this paper. The longitudinal displacement and rotation on both of the system ends are limited by the discrete elements in the form of translational and rotational springs. The results of numerical simulations concern the first vibration frequency (linear and non-linear components) in relation to the location and magnitude of external load application and different rotational spring stiffness. This nonlinear system is based on the screw drive used in the newly designed vertical platform lifts.
\end{abstract}

MSC 2010: 74H45, 65L10

Keywords: slender nonlinear systems, amplitude of vibrations, Euler's load

\section{Introduction}

Geometrically nonlinear systems (columns, beams) are the subjects of many studies [1-3] in which the nonlinear theory and the theory of Bernoulli-Euler are used to formulate the boundary problem. This theory is sufficient when slender systems are considered and when the system is combined with translational and rotational inertia of mass elements. The second possibility is to apply the theory of beams proposed by Timoshenko, which takes into account the shear energy and the rotational inertia energy of the cross section [4-6]. One of the more interesting non-linear structures is the partially tensioned column which was presented in works [7-11]. The publications show the crucial influence of discrete elements on the vibration frequency $[2,9]$ and stability [8] (the natural vibration frequency and critical force of the system can be controlled). The main scope of the study in [11] was to investigate the influence of the rotational stiffness of the support on the linear component of vibration frequency which does not depend on amplitude. The results of this study showed that the great impact of rotational stiffness on the linear compo- 
nent of vibrations at different locations of external load application and magnitude. With the increase in the rotational stiffness of the fastening points the vibration frequency of the system grows. Greater external load magnitude results in a more dynamic response of the investigated parameter. A significant influence of the amplitude on the natural vibration frequency was presented in [10], where the considered system was a partially tensioned rod rigidly fixed at both ends.

In this paper the influence of parameters such as translational and rotational stiffness of used springs and the external load magnitude for a variable location on the vibration frequency (nonlinear component) is presented. The column in question corresponds to a screw drive system, which is used in vertical lift platforms (equipped with an engine room located in the lower part of the frame).

\section{Boundary problem}

An external force $P$ is applied between the ends of the slender structure shown in Figure 1. Euler's load subjected in point $O$ does not change the line of action during the system deflection. The point of force application (described with parameter $\zeta$ ) can be changed along the entire length of the rod as when the screw-drive lift platform transports different loads at various lifting heights. In order to formulate a mathematical model, the overall length of the system is divided into two parts, respectively of lengths $l_{1}$ and $l_{2}$ (the compressed lower part is indicated by index 1 , and the tensioned upper part by index 2$)$. The considered structure is characterized (over the whole length) by constant bending stiffness $\left((E J)_{1}=(E J)_{2}=(E J)\right)$, longitudinal stiffness $\left((E A)_{1}=(E A)_{2}=(E A)\right)$ and mass per length unit $\left((\rho A)_{1}=(\rho A)_{2}=\right.$ $=(\rho A)$ ) (where: $E_{i}$ - Young's modulus, $\rho_{i}$ - density, $A_{i}$ - cross-section area, $J_{i}$ - geometrical axial moment of inertia of the cross-section of $i$-th element of the structure). The longitudinal displacement and rotation on both of the system ends are limited by the discrete elements in the form of translational $\left(K_{0}, K_{1}\right)$ and rotational $\left(C_{0}, C_{1}\right)$ springs.

The differential equations of motion and natural boundary conditions of the considered structure have been formulated on the basis of Hamilton's principle and the Bernoulli-Euler theory. The differential equations of motion in transversal and longitudinal directions of the system are as follows:

$$
\begin{aligned}
& \frac{\partial^{4} w_{i}\left(\xi_{i}, \tau\right)}{\partial \xi_{i}^{4}}+k_{i}^{2}(\tau) \frac{\partial^{2} w_{i}\left(\xi_{i}, \tau\right)}{\partial \xi_{i}^{2}}+\Omega_{i}^{2} \frac{\partial^{2} w_{i}\left(\xi_{i}, \tau\right)}{\partial \tau^{2}}=0 \\
& u_{i}\left(\xi_{i}, \tau\right)-u_{i}(0, \tau)=-\frac{k_{i}^{2}(\tau)}{\theta_{i}} \xi_{i}-\frac{1}{2} \int_{0}^{\xi_{i}}\left(\frac{\partial w_{i}\left(\xi_{i}\right)}{\xi_{i}}\right)^{2} d \xi_{i}
\end{aligned}
$$




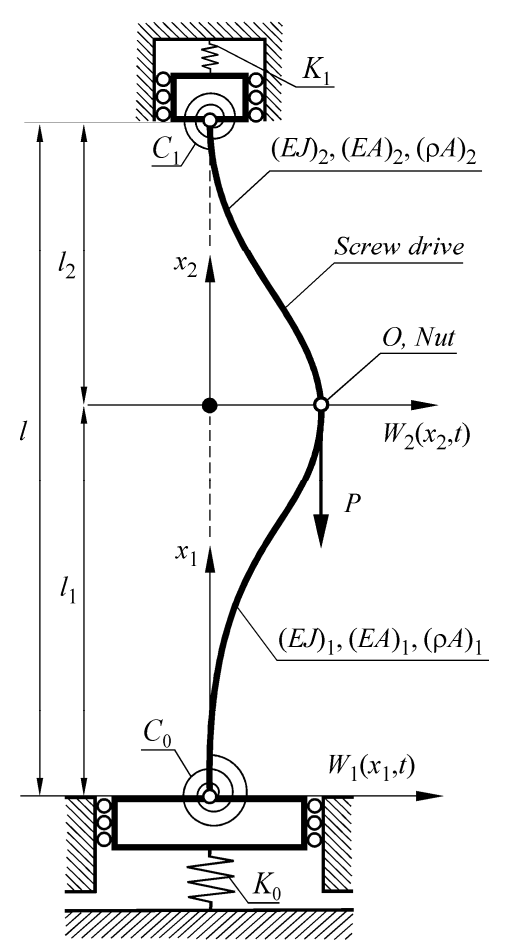

Fig. 1. Physical model of considered column

Equations (1) and (2) are written in the non-dimensional form using the following relations:

$$
\begin{gathered}
\xi_{i}=\frac{x_{i}}{l} ; \quad \zeta_{i}=\frac{l_{i}}{l} ; \quad w_{i}\left(\xi_{i}, \tau\right)=\frac{W_{i}\left(x_{i}, t\right)}{l} ; \quad u_{i}\left(\xi_{i}, \tau\right)=\frac{U_{i}\left(x_{i}, t\right)}{l} \\
k_{i}^{2}(\tau)=\frac{S_{i}(\tau) l^{2}}{(E J)_{i}} ; \Omega_{i}^{2}=\frac{(\rho A)_{i} \omega^{2} l^{4}}{(E J)_{i}} ; \tau=\omega t ; \quad \Theta_{i}=\frac{A_{i} l^{2}}{J_{i}} ; i=1,2
\end{gathered}
$$

where: $S_{i}(\tau)$ - internal force in $i$-th element of the structure, $\omega$ - vibration frequency, $W_{i}\left(x_{i}, \tau\right), U_{i}\left(x_{i}, \tau\right)$ - transversal and longitudinal displacements.

The geometrical and natural boundary conditions are presented below:

$$
\begin{gathered}
w_{1}\left(\zeta_{1}, \tau\right)=w_{2}(0, \tau) ;\left.\quad w_{1}^{I}\left(\xi_{1}, \tau\right)\right|^{\xi_{1}=\zeta_{1}}=\left.w_{2}^{I}\left(\xi_{2}, \tau\right)\right|_{\xi_{2}=0} ; \quad u_{1}\left(\zeta_{1}, \tau\right)=u_{2}(0, \tau) ; \\
w_{1}(0, \tau)=w_{2}\left(\zeta_{2}, \tau\right)=0 ; \\
\left.(E J)_{1} w_{1}^{I I}\left(\xi_{1}, \tau\right)\right|_{\xi_{1}=0} \frac{1}{l}-\left.C_{0} w_{1}^{I}\left(\xi_{1}, \tau\right)\right|_{\xi_{1}=0}=0 ; k_{1}^{2}(\tau) \frac{(E J)_{1}}{l^{2}}-K_{0} u_{1}\left(\zeta_{1}, \tau\right) l=0 ; \\
-\left.(E J)_{2} w_{2}^{I I}\left(\xi_{2}, \tau\right)\right|^{\xi_{2}=\zeta_{2}} \frac{1}{l}-\left.C_{1} w_{2}^{I}\left(\xi_{2}, \tau\right)\right|^{\xi_{2}=\zeta_{2}}=0 ; \quad k_{2}^{2}(\tau) \frac{(E J)_{2}}{l^{2}}-K_{1} u_{2}\left(\zeta_{2}, \tau\right) l=0 ;
\end{gathered}
$$




$$
\begin{gathered}
\left.(E J)_{1} w_{1}^{I I}\left(\xi_{1}, \tau\right)\right|^{\xi_{1}=\zeta_{1}}-\left.(E J)_{2} w_{2}^{I I}\left(\xi_{2}, \tau\right)\right|_{\xi_{2}=0}=0 \\
\left.(E J)_{1} w_{1}^{I I I}\left(\xi_{1}, \tau\right)\right|^{\xi_{1}=\zeta_{1}}-\left.(E J)_{2} w_{2}^{I I I}\left(\xi_{2}, \tau\right)\right|_{\xi_{2}=0}+\left.P l^{2} w_{2}^{I}\left(\xi_{2}, \tau\right)\right|_{\xi_{2}=0}=0 \\
k_{1}^{2}(\tau) \frac{(E J)_{1}}{l^{2}}-k_{2}^{2}(\tau) \frac{(E J)_{2}}{l^{2}}-P=0
\end{gathered}
$$

The nonlinear elements of the differential equations and boundary conditions are expanded into a power series of the small parameter of an amplitude. The small parameter method is used because of nonlinearities of the solution of the boundary problem of free vibration. This investigation focuses only on the rectilinear form of static equilibrium at which the series is as follows:

$$
\begin{gathered}
w_{i}(\xi, \tau)=\sum_{j=1}^{N} \varepsilon^{2 j-1} w_{i 2 j-1}(\xi, \tau)+O\left(\varepsilon^{2(N+1)}\right) \\
u_{i}(\xi, \tau)=u_{i 0}(\xi)+\sum_{j=1}^{N} \varepsilon^{2 j} u_{i 2 j}(\xi, \tau)+O\left(\varepsilon^{2(N+1)}\right) \\
k_{i}^{2}(\tau)=k_{i 0}^{2}+\sum_{j=1}^{N} \varepsilon^{2 j} k_{i 2 j}^{2}(\tau)+O\left(\varepsilon^{2(N+1)}\right) \\
\Omega_{i}^{2}=\Omega_{i 0}^{2}+\sum_{j=1}^{N} \varepsilon^{2 j} \Omega_{i 2 j}^{2}+O\left(\varepsilon^{2(N+1)}\right) \\
\omega^{2}=\omega_{0}^{2}+\sum_{j=1}^{N} \varepsilon^{2 j} \omega_{2 j}^{2}+O\left(\varepsilon^{2(N+1)}\right)
\end{gathered}
$$

where:

$$
\begin{aligned}
& w_{i 1}(\xi, \tau)={ }^{(1)} w_{i 1}(\xi) \cos \tau ; \quad w_{i 3}(\xi, \tau)={ }^{(1)} w_{i 3}(\xi) \cos \tau+{ }^{(3)} w_{i 3}(\xi) \cos 3 \tau ; \ldots \\
& u_{i 2}(\xi, \tau)=u_{i 2}^{(0)}(\xi)+u_{i 2}^{(2)}(\xi) \cos 2 \tau ; \ldots \\
& \text { (0) (2) } \\
& k_{i 2}^{2}(\tau)=k_{i 2}^{2}+k_{i 2}^{2} \cos 2 \tau ; \ldots
\end{aligned}
$$

Solutions of equations (1), taking into account a power series of the small parameter, are substituted with the boundary conditions associated with transverse deflection, resulting in a system of equations. The determinant of this system equalized to zero is a transcendental equation on the linear component of the natural vibration frequency $\omega_{0}$. The nonlinear component of the natural vibration frequency $\omega_{2}$ is calculated by the following formula: 


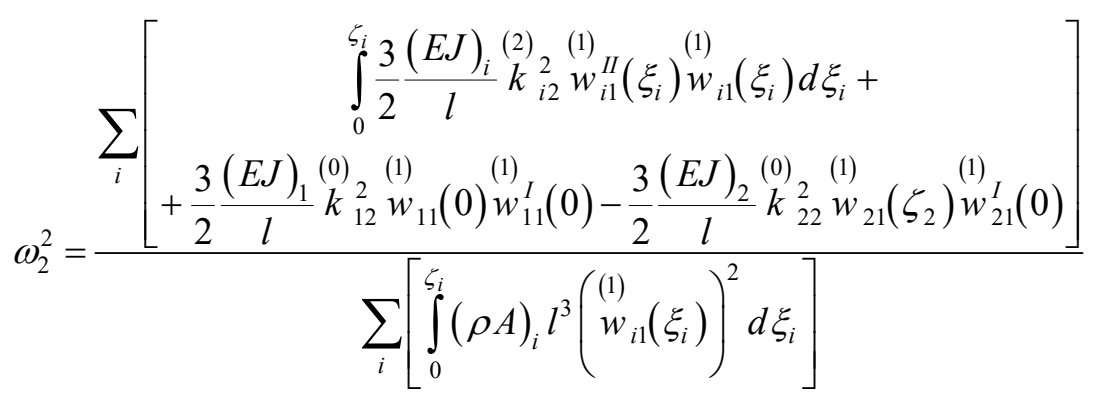

where:

$$
\stackrel{(0)}{k}{ }_{22}^{2}=-\frac{\frac{1}{4} \sum_{i} \int_{0}^{\zeta_{i}}\left({ }^{(1)} w_{i 1}^{I}\left(\xi_{i}\right)\right)^{2} d \xi_{i}}{\frac{(E J)_{2}}{l^{3} K_{1}}+\frac{(E J)_{2}}{l^{3} K_{0}}+\frac{1}{\Theta_{1}} \frac{(E J)_{2} \zeta_{1}}{(E J)_{1}}+\frac{\zeta_{2}}{\Theta_{2}}} ; \stackrel{(0)}{k}{ }_{12}^{2}=\stackrel{(0)}{k} \underset{22}{2} \frac{(E J)_{2}}{(E J)_{1}}
$$

(1) $w_{i 1}\left(\xi_{i}\right)$ are solutions for differential equations associated with a small parameter in zero power. These solutions were obtained on the basis of a normalization condition.

\section{Results of numerical simulations}

Results of numerical simulations of free vibrations (including linear $\omega_{0}$ and nonlinear $\omega_{2}$ components of free vibrations frequency) of the considered partially tensioned slender system were presented in non-dimensional form, defined as:

$$
\lambda=\frac{P l^{2}}{(E J)_{1}} ; \quad c_{j}=\frac{C_{j} l}{(E J)_{1}} ; \quad k_{j}=\frac{K_{j} l^{3}}{(E J)_{1}} ; \quad \zeta=\frac{l_{1}}{l} ; \quad \zeta_{\omega}=\frac{\omega-\omega_{0}}{\omega_{0}} 100 \% ; j=0,1
$$

The amplitude of vibrations in numerical calculations is assumed as a double radius of gyration. In Figure 2 the relationship between the $\zeta_{\omega}$ parameter (difference between the nonlinear and linear free vibration frequency) and the $\zeta$ parameter (location of Euler's load application) in combination for different rotational spring stiffness is plotted. In simulations it has been assumed that external load magnitude $\lambda=10$, translational stiffness is $k_{0}=k_{1}=1000$ (Fig. 2a), $k_{0}=k_{1}=2000000$ (Fig. 2b) and rotational stiffness $c_{0}=c_{1}$. The influence of the rotational spring stiffness on dynamic behavior was analyzed. On the basis of the obtained results, it can be concluded that the rotational stiffness has great influence on the magnitude of the nonlinear component vibration frequency. With an increase in the rotational stiffness of the fastening points, the share of the nonlinear component of the vibration 
frequency decreases. Conversely, in the case of increased translational stiffness (Fig. $2 \mathrm{~b}$ ), much higher values of the $\zeta_{\omega}$ parameter are obtained. The difference between the nonlinear and linear free vibration frequency changes in relation to the location of the external force $\zeta$ at a given rotational stiffness.
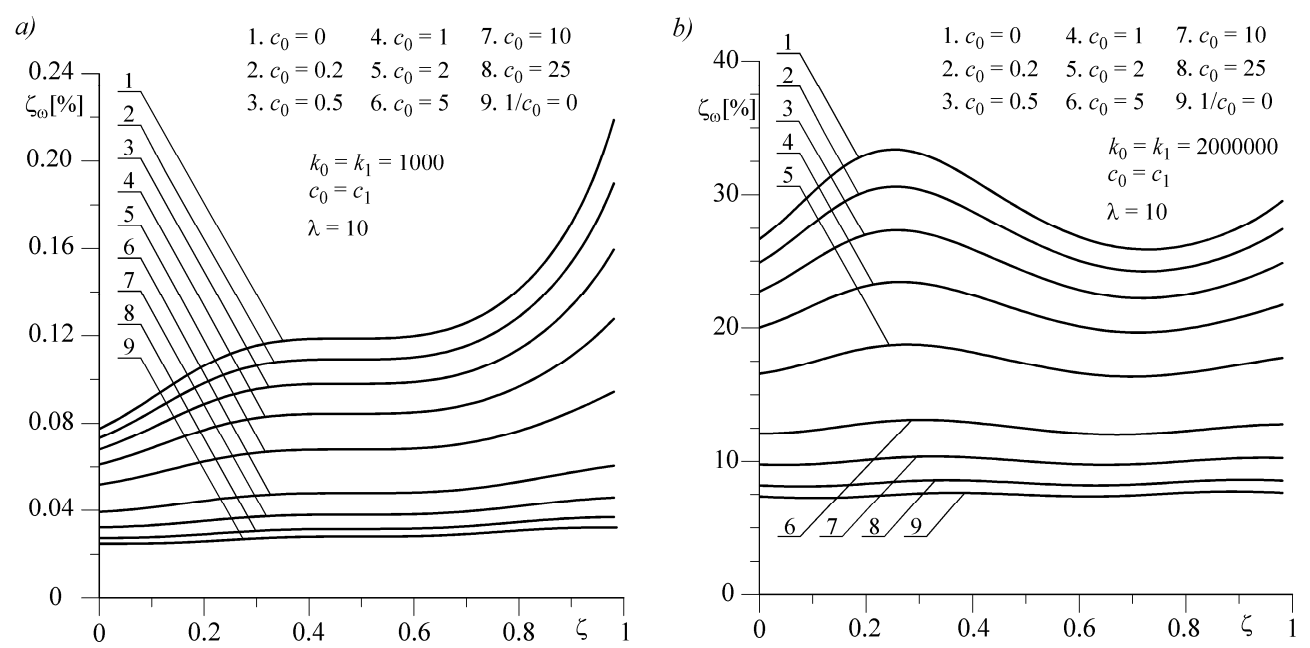

Fig. 2a-b. The difference between the nonlinear and linear components of vibration frequency $\zeta_{\omega}$ in relation to the point of external load application $\zeta$ at different rotational spring stiffness and taking into account parameters $\lambda=10, c_{0}=c_{1}, k_{0}=k_{1}$
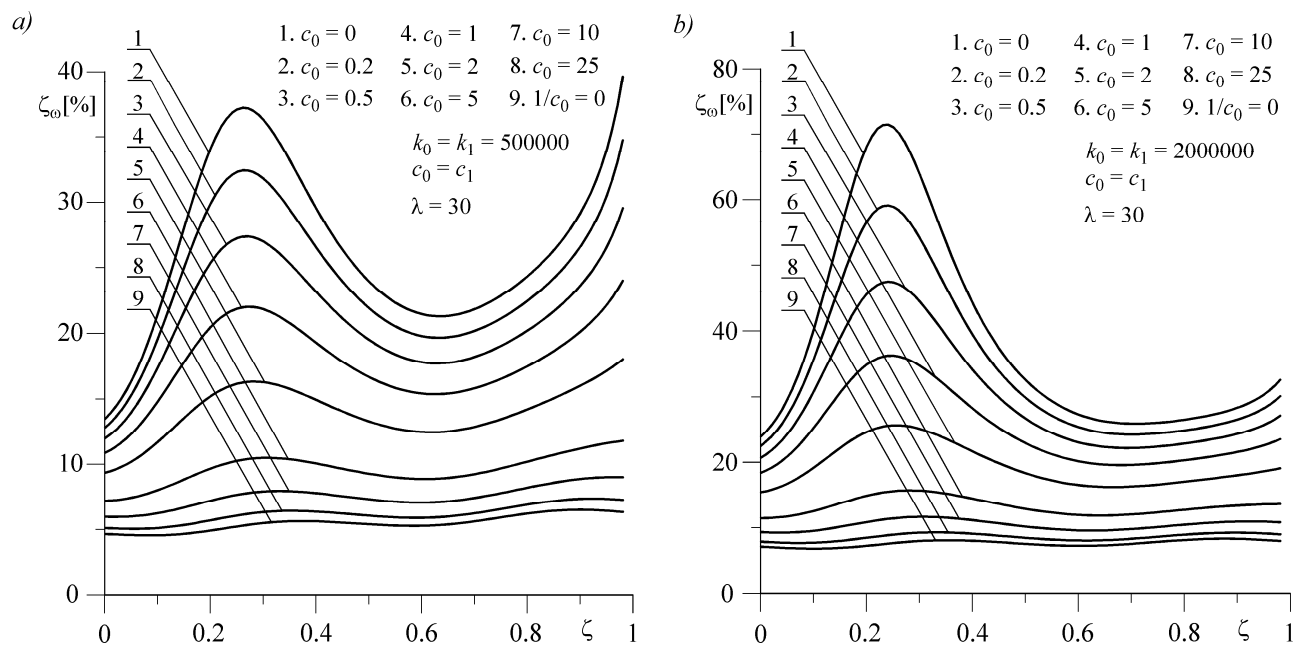

Fig. 3a-b. The difference between the nonlinear and linear components of vibration frequency $\zeta_{\omega}$ in relation to the point of external load application $\zeta$ at different rotational spring stiffness and taking into account parameters $\lambda=30, c_{0}=c_{1}, k_{0}=k_{1}$

For a higher magnitude of the external load $\lambda=30$, greater changes of the $\zeta_{\omega}$ parameter were obtained, which depended on the point of load application, as shown 
in Figure 3. It can also be observed that the values of the parameter of the difference between the linear and the nonlinear free vibration frequency are greater when the point of external load application is $\zeta=0.25$ and when it is located near the upper mounting of the column. The obtained results will contribute to the correct selection of rigidity of the mounting screw drive of the vertical platform lift to avoid the frequency of resonance vibrations. The elevator is designed for disabled people and the safety of use is very important, making it difficult to change the magnitude of external load $(\lambda)$ and the lifting height $(l)$.

\section{Conclusions}

The boundary problem of free vibrations of a partially tensioned system subjected to Euler's load was considered in this paper. The influence of the rotational spring stiffness on dynamic behavior was analyzed. The discussion includes both a linear component of the first vibration frequency as well as its nonlinear component, the magnitude of which depends on the amplitude of vibration. When there is an increase in the rotational stiffness of the fastening points, the vibration frequency of the system increases, while the share of the nonlinear component of the vibration frequency decreases. In contrast, in the case of increasing translational stiffness of the mountings, the nonlinear component of the vibration frequency increases. On the basis of the obtained results, it can be concluded that the vibration frequency of the considered slender system can be controlled by the stiffness of the used rotational $\left(C_{0}, C_{1}\right)$ and translational $\left(K_{0}, K_{1}\right)$ springs and magnitude of the external load.

\section{Acknowledgement}

The study has been carried out within the statutory funds of the Czestochowa University of Technology BS/PB 1-101/3020/17/P and BS/MN 1-101/305/18/P.

\section{References}

[1] Awrejcewicz, J., Saltykova, O.A., Chebotyrevskiy, Yu.B., \& Krysko, V.A. (2011). Nonlinear vibrations of the Euler-Bernoulli beam subjected to transversal load and impact actions. Nonlinear Studies, 18(3), 329-364.

[2] Sundararajan, C. (1976). Influence of an elastic end support on the vibration and stability of Beck's column. International Journal of Mechanical Sciences, 18, 239-241.

[3] Przybylski, J., \& Tomski, L. (1992). Vibration of an Initially Prestressed Compound Column under Axial Compression. Elsevier Science Publishers B.V., 263-268.

[4] Jiang, L.Y., \& Yan, Z. (2010). Timoshenko beam model for static bending of nanowires with surface effects. Physica E, 42, 2274-2279.

[5] Abramovich, H., \& Hamburger, O. (1991). Vibration of a cantilever Timoshenko beam with a tip mass. Journal of Sound and Vibration, 148(1), 162-170.

[6] Nemat-Nasser, S. (1967). Instability of a cantilever under a follower force according to Timoshenko beam theory. Journal of Applied Mechanics, 484-485. 
[7] Uzny, S., Sokół, K., Osadnik, M. (2016). Free vibrations of the partially tensioned geometrically non-linear system subjected to Euler's load. Vibrations in Physical Systems, 27, 399-406.

[8] Uzny, S., \& Osadnik, M. (2017). Influence of longitudinal elastic support on stability of a partially tensioned column. Book Series: Engineering Mechanics, 1006-1009.

[9] Uzny, S., \& Osadnik, M. (2017). Influence of the longitudinal spring element on free vibrations of the partially tensioned column. Procedia Engineering, 17, 135-140.

[10] Uzny, S., Sokół, K., \& Osadnik, M. (2016). Influence of amplitude on free vibration frequency of a partially tensioned column. Book Series: Engineering Mechanics, 562-565.

[11] Uzny, S., \& Osadnik M. (2018). Influence of rotational stiffness of support of a partially tensioned column on its free vibrations. Book Series: Engineering Mechanics, 889-992. 